Süleyman Demirel Üniversitesi Fen Edebiyat Fakültesi Fen Dergisi

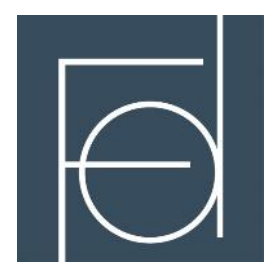

Süleyman Demirel University Faculty of Arts and Sciences Journal of Science

2019, 14(2): 327-334

DOI: $10.29233 /$ sdufeffd.605430

Atıf için / For Citation: İ. H. SARPÜN, A. İNAL, B. ÇEÇEN, "Voltaj ve Akım Değerlerinin Hasta Dozu Üzerindeki Etkilerinin CTDI Fantomu ile Araştırılması", Süleyman Demirel Üniversitesi Fen Edebiyat Fakültesi Fen Dergisi, 14(2), 327-334, 2019.

\title{
Voltaj ve Akım Değerlerinin Hasta Dozu Üzerindeki Etkilerinin CTDI Fantomu ile Araştırılması
}

\author{
İsmail Hakk1 SARPÜN ${ }^{* 1,2}$, Aysun İNAL ${ }^{3}$, Buket ÇEÇEN ${ }^{1}$ \\ ${ }^{1}$ Akdeniz Üniversitesi, Fen Fakültesi, Fizik Bölümü, 07057, Antalya, Türkiye \\ ${ }^{2}$ Akdeniz Üniversitesi, Nükleer Bilimler Uyg. ve Araş. Merkezi, 07057, Antalya, Türkiye \\ ${ }^{3}$ Sağllk Bilimleri Üniversitesi, Antalya Eğitim Araşttrma Hastanesi, Radyasyon Onkolojisi, 07100, \\ Antalya, Türkiye \\ *yazışllan yazar e-posta: isarpun@ gmail.com
}

(Alınış / Received: 15.08.2019, Kabul / Accepted: 11.10.2019, Yayımlanma / Published: 30.11.2019)

Özet: Bilgisayarlı tomografi (CT) günümüzde etkin bir şekilde kullanılan modern bir cihaz olup, tanısal görüntülemede çok önemli bir yer tutmaktadır. Bu çalıșmanın amacı, CT'de tüp voltajı (kVp), tüp akımı (mAs) ve kesit kalınlığı değerlerini değiştirerek farklı çaplara sahip biri $16 \mathrm{~cm}$ çapında ve diğeri $32 \mathrm{~cm}$ çapında olan su eşdeğeri silindirik iki adet fantomda doz değişimlerini incelemektir. İyonizasyon odası ile okunan soğurulan doz değerleri bilgisayarda var olan bir paket programı yardımıyla ilgili parametrelere çevrilmiştir. Belli bir hacim için hesaplanan $\mathrm{CTDI}_{\mathrm{vol}}$ ve kesit alınan uzunluk boyunca aldığı toplam doz değerini veren DLP değerleri karşılaştırılmıştır. Çalışma sonuçlarından da görüldügü gibi, doz artışı akım ve voltaj değerini arttırdıkça yükselmektedir. Özellikle kafa fantomu örneğindeki gibi küçük ve zayıf hastalarda doz artışı daha fazladır.

Anahtar kelimeler: CT, CTDI, Doz, Fantom

\section{Effects of Voltage and Current Values to Patient Dose by CTDI Phantom}

Abstract: Computed tomography is a modern device and plays an important role in diagnostic imaging. The aim of this study was to examine the dose changes in two water-equivalent cylindrical phantoms with different diameters, one $16 \mathrm{~cm}$ in diameter and the other $32 \mathrm{~cm}$ in diameter, by changing the tube voltage $(\mathrm{kVp})$, tube current $(\mathrm{mU})$ and slices thickness values in $\mathrm{CT}$. The absorbed dose values obtained by the ionization chamber were converted to the relevant parameters by means of a package program available on the computer. The calculated $\mathrm{CTDI}_{\text {vol }}$ for a given volume and the DLP values giving the total dose value taken during the cross-sectional length were compared. As can be seen from the results of the study, the dose increases with increasing the current and voltage values. Especially in small and thin patients such as the head phantom, the dose increase is higher.

Key words: CT, CTDI, Dose, Phantom 


\section{Giriş}

Bilgisayarlı tomografi (CT) gibi modern tanısal görüntüleme, tıpta çok önemli bir yer tutmaktadır. Bilgisayarlı tomografi birkaç saniye içinde vücudun herhangi bir bölümünün son derece ayrıntılı görüntülerini üretebilir. CT en sık kullanılan tanısal görüntüleme yöntemlerinden biridir fakat CT'den alınan iyonlaştırıcı radyasyon dozları, toraks röntgeni veya mamografi gibi standart bir röntgenin 50 ile 500 katı kadar olabilir [1]. Bu kadar yüksek radyasyon dozlarının yanı sıra, bu tanı prosedürlerinin yaygın ve artan kullanımın küçük ama önemli bir şekilde genel popülasyonda kanser riski oluşturabileceği endişesi doğmuştur. Bildirilen radyasyona aşırı maruz kalma olayları, CT incelemelerinde verilen dozların değerlendirilmesine olan ilgiye neden olmuştur [2].

BT'de radyasyon dozunun değerlendirilmesi, X-1şını kaynağının tek bir dönüşü sırasında ölçülen bilgisayarlı tomografi doz endeksini (CTDI) tahmin ederek yapılır. Bu endeks, CT tarayıcısının boyuna ekseni boyunca absorbe edilen dozu temsil etmektedir $[3,4]$. Doz ölçümleri, fantomun merkezinde ve periferinde gerçekleştirilir ve bu değerler, fantoma tek bir radyasyon dozu tahmini üretmek için ağırlıklı bir toplam değer $\left(\mathrm{CTDI}_{\mathrm{w}}\right)$ kullanılarak birleştirilir. Piyasada var olan kafa ve vücut fantomları sırasıyla $16 \mathrm{~cm}$ ve $32 \mathrm{~cm}$ çapındadır. Kafa fantomda ölçülen hacimsel bilgisayarlı tomografi doz endeksi $\mathrm{CTDI}_{\mathrm{vol}}$, kafa CT için bir referans olarak ve ayrıca bazı tarayıcı üreticileri için pediatrik vücut $\mathrm{CT}$ için bir referans olarak kullanılırken vücut fantomunda ölçüldüğünde yetişkinler için referans olarak kullanılır. CTDI $_{\mathrm{vol}}$ birimi mGy'dir.

Yeni nesil CT tarayıcılarla, helikal teknolojili ileri teknolojiler tarama modu veya conebeam 1şınlama geometrileri, doz tahmin yöntemi Amerikan Tıp Fizikçiler Birliği (AAPM) Görev Grubu (TG) raporlarında belirtildiği şekilde gelişmeye devam etmektedir [3-5]. Genel olarak, doz dengesini sağlamak için bir fantoma yerleştirilmiş küçük hacimli bir iyonizasyon odasının kullanımı, absorbe edilen dozu tahmin etmek için önerilmektedir [5].

$\mathrm{Bu}$ çalışmanın amacı, CT’de tüp voltajı $(\mathrm{kVp})$, tüp akımı (mAs) ve kesit kalınlığı değerlerini değiştirerek farklı çaplara sahip iki fantomda doz değişimlerini incelemektir.

\section{Materyal ve Metot}

Çalışmada, biri $16 \mathrm{~cm}$ çapında (kafa fantomu) ve diğeri $32 \mathrm{~cm}$ çapında vücut fantomu) olan su eşdeğeri silindirik iki adet fantom kullanılmıştır. Ölçümler, Bilgisayarlı Tomografide (GE-Optima580, GE, USA), 4 farklı kesit kalığ $1(0,625,2,5,5$ ve $10 \mathrm{~mm})$, 4 farklı tüp akımı (50, 100, 200 ve 400 mAs) ve 4 farklı Tüp voltajı $(80,100,120$ ve $140 \mathrm{kVp}$ ) değişimi yapılarak alınmıştır. Çalışmada pitch değeri 1 olarak kabul edilip tüm ölçümlerde bu değer kullanılmıştır.

Ölçümlerde, $100 \mathrm{~mm}$ uzunluğunda pencil iyonizasyon odası ve okuma değerlerinin gerçekleştirildiği X-1şını elektrometre (Piranha, RTI Electronics) kullanılmıştır. Okunan absorbe doz değerleri bilgisayarda var olan Ocean programı (RTI Electronics) vasıtasıyla ilgili parametrelere çevrilmiştir. Çalışmada, belli bir hacim için hesaplanan $\mathrm{CTDI}_{\text {vol }}$ ve kesit alınan uzunluk boyunca aldığı toplam doz değerini veren Dose Length Product (DLP) değerleri karşılaştırılmıştır. 
Yukarıda bahsi geçen parametreler programda var olan algoritma ile hesaplanmaktadır. Hesaplama sırasında aşağıda var olan denklemler ile CTDI $_{\text {vol ve DLP parametrelerinin }}$ hesaplaması gerçekleştirilmektedir. CT doz indeksini hesaplamanın merkezi ve periferik CTDI değerlerini ağırlıklı toplam $\left(\mathrm{CTDI}_{\mathrm{w}}\right)$ şeklinde ifade etmektir [6].

$$
C T D I_{w}=\frac{1}{3} C T D I_{100}^{A}+\frac{2}{3} C T D I_{100}^{B}
$$

Bilgisayarlı tomografi helikal bir tarama için pitch; X-1şını tüpünün bir dönüşü süresinde masanın mm cinsinden hareket miktarının, detektörün tarayıcının eş merkezine izdüşümü olan nominal ışınlama geniş̧liğine bölünmesiyle hesaplanır [6]. Helikal ölçümler için hacim $\mathrm{CTDI}_{\mathrm{vol}}$ değeri;

$$
C T D I_{v o l}=C T D I_{w} \frac{1}{p i t c h}
$$

Bilgisayarlı tomografi taramasında toplam soğurulan dozu hesaplamak için, taranan aralık dikkate alınmalıdır. Doz uzunluk çarpımı (DLP), CTDI ${ }_{\mathrm{vol}}$ ile tarama uzunluğunun (L) çarpımıdır. Çalışmada tüm ölçümler $150 \mathrm{~mm}$ boyunca alınmıştır. Doz uzunluk çarpımının birimi mGy.cm'dir [6].

$$
\mathrm{DLP}=C T D I_{v o l} \cdot \mathrm{L}=\left(\frac{1}{3} C T D I_{100}^{A}+\frac{2}{3} C T D I_{100}^{B}\right) \frac{1}{p i t c h} \cdot \mathrm{L}
$$

\section{Bulgular}

Kafa fantomu için kesit kalınlığı, tüp akımı ve tüp voltajına bağlı $\mathrm{CTDI}_{\mathrm{vol}}$ değişimi Tablo 1'de görülmektedir. Bulgulara göre, tüm voltaj ve akım değerlerinde kesit kalınlığına göre değişim 0,625-5 mm aralığında ortalama \%1,58 $(\% 0,45$ - \%8,85) olmak üzere, 0,625-10 mm aralığında ise ortalama \%13,2 artmıştır (\%4,9 - \%18,9). Tüp akımı değişimine göre $\mathrm{CTDI}_{\mathrm{vol}}$ değeri akım yükseldikçe artma göstermiştir. En fazla, tüp akım değeri 50 mAs'dan 400 mAs'ya yükseldiğinde $140 \mathrm{kVp}$ tüp voltaj1 ve $10 \mathrm{~mm}$ kesit kalınlığında $\mathrm{CTDI}_{\mathrm{vol}}$ değerinin 17,5 kat artmış olduğu hesaplanmıştır. Tüp voltajı arttıkça tüm kesit kalınlıkları ve tüp akım değerleri için doz değerlerinde artış gözlenmiştir. Voltaj değeri 80 kVp'den 140 kVp'ye yükseldiğinde 200 mAs'lik tüp akımı değeri ve $10 \mathrm{~mm}$ kesit kalınlığ 1 için $\mathrm{CTDI}_{\mathrm{vol}}$ değerinin yaklaşı 10 kat artmış olduğu hesaplanmıştır.

Tablo1. Kafa fantomu için tüp voltajı ve kesit kalınlığına göre CTDIvol (mGy) değerleri

\begin{tabular}{cccccc}
\hline & & \multicolumn{4}{c}{ CTDI $_{\text {vol }}$ (mGy) } \\
\cline { 2 - 6 } Tüp Akımı & $\begin{array}{c}\text { Kesit Kalınlığı } \\
(\mathbf{m m})\end{array}$ & \multicolumn{4}{c}{ Tüp Voltajı (kVp) } \\
\cline { 2 - 6 } & 0,625 & 1,57 & 2,99 & 4,43 & 6,81 \\
\cline { 2 - 6 } 50 mAs & 2,5 & 1,56 & 2,98 & 4,49 & 6,78 \\
\cline { 2 - 6 } & 5 & 1,56 & 3,04 & 4,50 & 6,91 \\
& 10 & 1,29 & 2,51 & 3,82 & 5,55 \\
\hline \multirow{2}{*}{$100 \mathrm{mAs}$} & 0,625 & 3,09 & 5,96 & 8,91 & 23,44 \\
\cline { 2 - 6 } & 2,5 & 3,07 & 6,01 & 8,89 & 23,22 \\
\hline \multirow{nyyyyy}{*}{$200 \mathrm{mAs}$} & 5 & 3,12 & 6,07 & 8,89 & 23,76 \\
\hline & 10 & 2,60 & 4,94 & 7,48 & 25,24 \\
\hline
\end{tabular}




\begin{tabular}{cccccc}
\hline & 2,5 & 6,14 & 11,91 & 17,94 & 47,03 \\
\cline { 2 - 5 } & 5 & 6,23 & 12,18 & 17,91 & 47,56 \\
\cline { 2 - 5 } & 10 & 4,99 & 9,96 & 14,93 & 50,53 \\
\hline \multirow{3}{*}{$400 \mathrm{mAs}$} & 0,625 & 21,55 & 22,16 & 39,02 & 90,43 \\
\cline { 2 - 5 } & 2,5 & 21,49 & 22,23 & 39,03 & 90,13 \\
\cline { 2 - 5 } & 5 & 21,91 & 24,13 & 39,21 & 91,20 \\
\cline { 2 - 6 } & 10 & 20,45 & 19,78 & 31,96 & 95,29
\end{tabular}

Vücut fantomu için kesit kalınlığı, tüp akımı ve tüp voltajına bağlı $\mathrm{CTDI}_{\mathrm{vol}}$ değişimi Tablo 2'de görülmektedir. Bulgulara göre, tüm voltaj ve akım değerlerinde kesit kalınlığına göre değişim 0,625-5 $\mathrm{mm}$ aralığında ortalama \%1,93 (Minimum ve maksimum: $\% 0,81$ ve \%8,0) olmak üzere, 0,625-10 $\mathrm{mm}$ için $\mathrm{CTDI}_{\mathrm{vol}}$ değeri fark1 ortalama \%12,8 artmıştır. (Minimum ve maksimum: \%5,4 ve \%18,45). Tüp akımı değişimine göre $\mathrm{CTDI}_{\mathrm{vol}}$ değeri akım yükseldikçe artma göstermiştir. Tüp akım değeri 50 mAs'dan 400 mAs'ya yükseldiğinde $140 \mathrm{kVp}$ tüp voltaj1 ve $10 \mathrm{~mm}$ kesit kalınlığında CTDI $_{\text {vol }}$ değerinin 17,2 kat artmış olduğu hesaplanmıştır. Tüp voltajı arttıkça tüm kesit kalınlıkları ve tüp akım değerlerinde artış gözlenmiştir. Voltaj değeri 80 kVp'den 140 kVp'ye yükseldiğinde $\mathrm{CTDI}_{\mathrm{vol}}$ değerinin 2,9 kat artmış olduğu hesaplanmıştır.

Tablo2. Vücut fantomu için tüp voltajı ve kesit kalınlığına göre CTDIvol (mGy) değerleri

\begin{tabular}{|c|c|c|c|}
\hline \multirow[b]{2}{*}{ Tüp Akımı } & \multirow{2}{*}{$\begin{array}{c}\text { Kesit Kalınlığı } \\
(\mathbf{m m})\end{array}$} & \multicolumn{2}{|c|}{$\frac{\text { CTDI }_{\text {vol }}(\mathrm{mGy})}{\text { Tüp Voltajı }(\mathrm{kVp})}$} \\
\hline & & 80 & 140 \\
\hline \multirow{4}{*}{$50 \mathrm{mAs}$} & 0,625 & 0,868 & 1,111 \\
\hline & 2,5 & 0,865 & 1,106 \\
\hline & 5 & 0,875 & 1,127 \\
\hline & 10 & 0,721 & 0,906 \\
\hline \multirow{4}{*}{$100 \mathrm{mAs}$} & 0,625 & 1,729 & 3,826 \\
\hline & 2,5 & 1,744 & 3,790 \\
\hline & 5 & 1,747 & 3,878 \\
\hline & 10 & 1,42 & 4,119 \\
\hline \multirow{4}{*}{$200 \mathrm{mAs}$} & 0,625 & 3,469 & 7,689 \\
\hline & 2,5 & 3,454 & 7,676 \\
\hline & 5 & 3,504 & 7,762 \\
\hline & 10 & 2,864 & 8,247 \\
\hline \multirow{4}{*}{$400 \mathrm{mAs}$} & 0,625 & 6,428 & 14,759 \\
\hline & 2,5 & 6,448 & 14,710 \\
\hline & 5 & 6,941 & 14,885 \\
\hline & 10 & 5,69 & 15,552 \\
\hline
\end{tabular}

Kesit kalınlığ1 ve tüp akımına göre DLP değişimleri 80, 100, 120 ve $140 \mathrm{kVp}$ için sırasıyla Şekil 1, 2, 3 ve 4'de görülmektedir. Tüp akımı arttıkça DLP değeri artmıștır. En büyük artış $10 \mathrm{~mm}$ kesit kalınlığında, $80 \mathrm{kVp}$ tüp voltajında $50 \mathrm{mAs}$ ile $400 \mathrm{mAs}$ arasında 17,5 kat olarak bulunmuştur. 


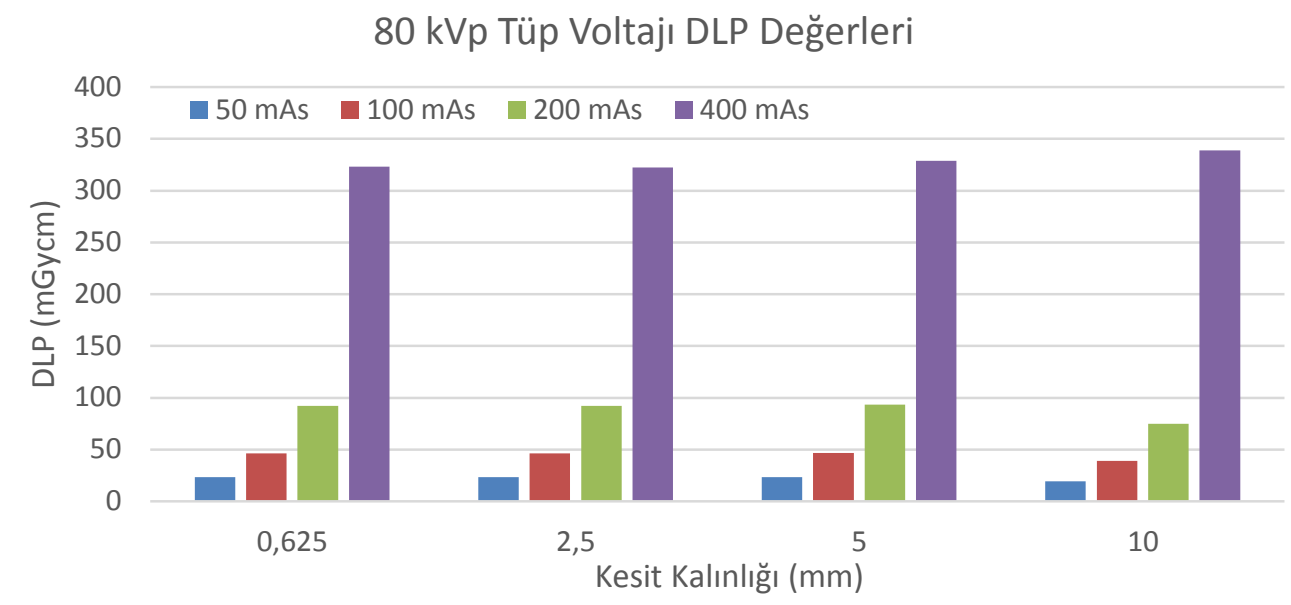

Şekil 1. 80 kVp için Tüp akımı ve kesit kalınlığına göre DLP değerlerinin değişim grafiği

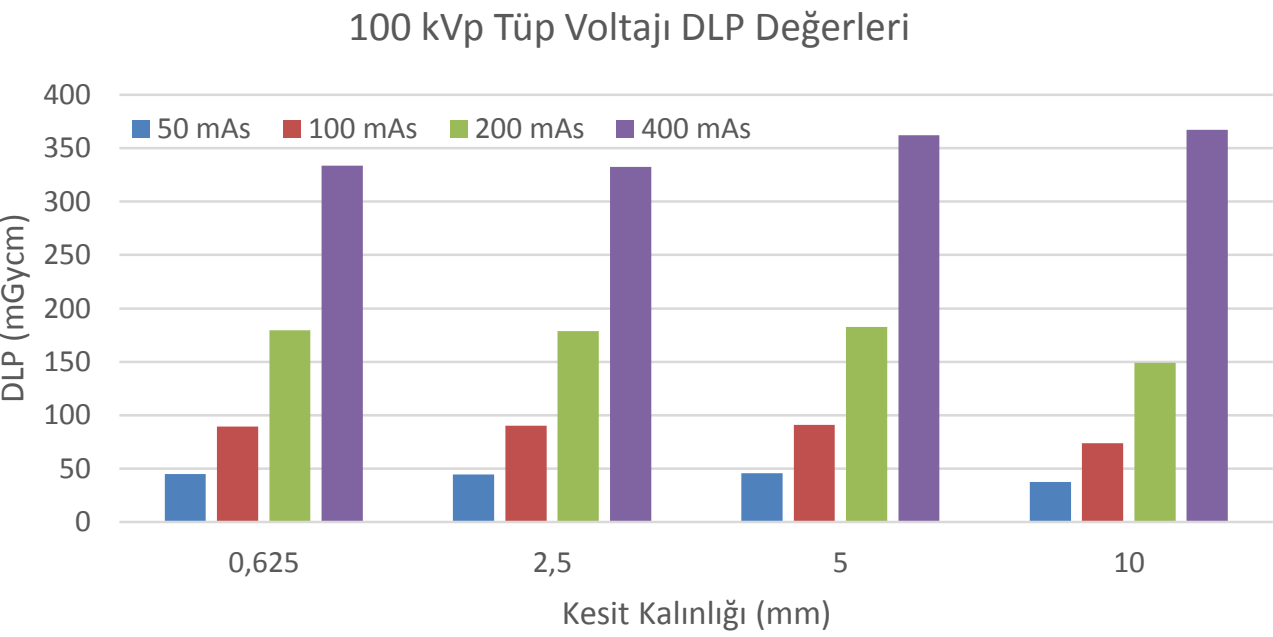

Şekil 2. $1000 \mathrm{kVp}$ için Tüp akımı ve kesit kalınlığına göre DLP değerlerinin değişim grafiğgi

\section{0 kVp Tüp Voltajı DLP Değerleri}

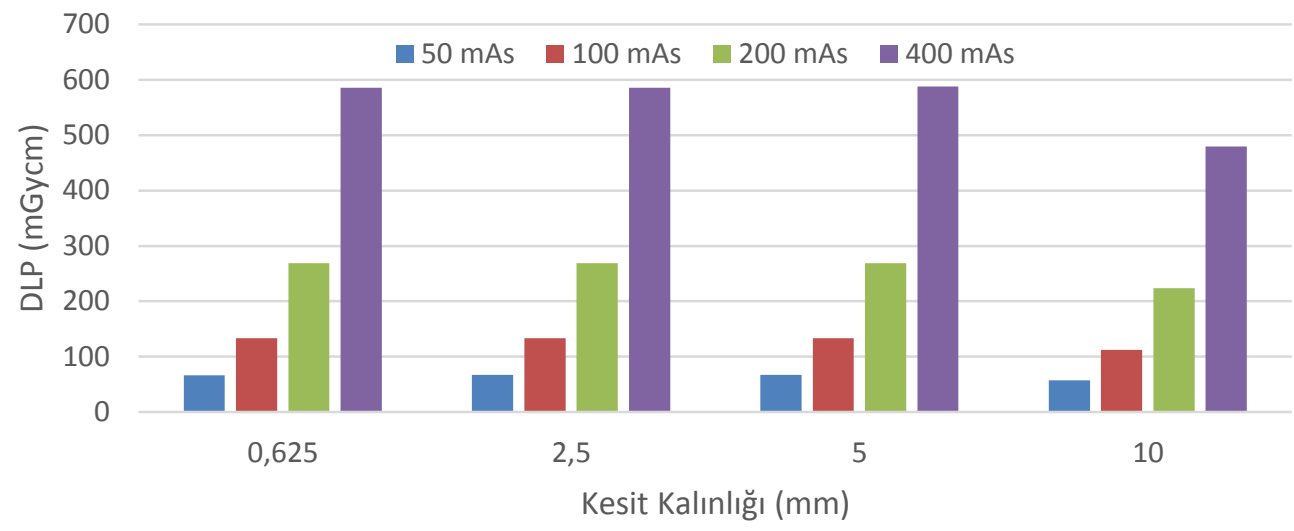

Şekil 3. $1200 \mathrm{kVp}$ için Tüp akımı ve kesit kalınlığına göre DLP değerlerinin değişim grafiği 


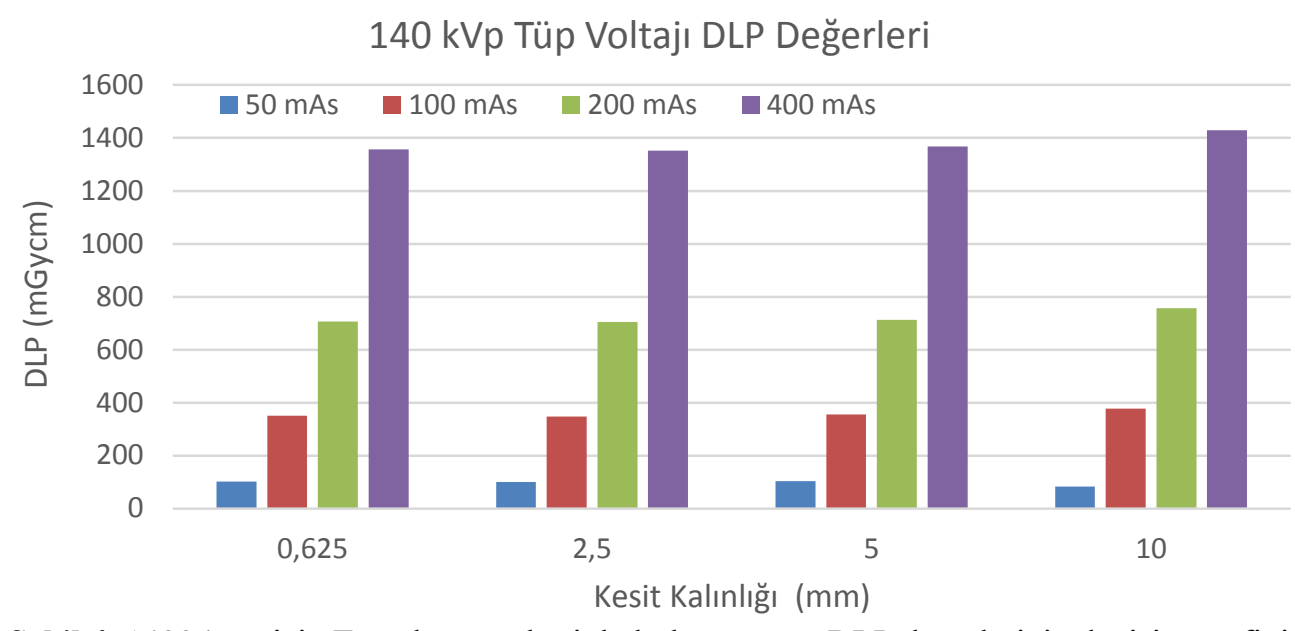

Şekil 4. 1400 kVp için Tüp akımı ve kesit kalınlığına göre DLP değerlerinin değişim grafiği

\section{Sonuç ve Yorum}

$\mathrm{CTDI}_{\mathrm{vol}}$, kullanıcıların saçılan radyasyon miktarını ölçmelerini ve farklı tarama protokolleri veya tarayıcıları arasındaki radyasyon çıkışını karşılaştırmasını sağlayan, silindirik bir akrilik fantomla ölçülen radyasyon dozunun standart ölçü parametresidir. Fakat, CT'den çıkan dozu, hasta dozu ile eşleştirmek için hastanın boyutu, ışınlanmış organlar, vücut kompozisyonu ve tarama aralığ1 dikkate alınarak karmaşık hesaplamalar gerekir [7-9]. Çoğu çalışma, tüp voltajı değerinin düşürülmesi, genel doz üzerinde tüp akımı değerinin düşürülmesinden daha büyük bir etkiye sahip olduğundan; hasta dozunu en aza indirmek için tüp voltajını azaltmaya ( $80 \mathrm{kVp}$ bölgesine kadar) odaklanır [10-13].

Çalışmalar sonucu, kontrastlı çekimler, çocuklar ve zayıf hastalar için düşük tüp voltajı önerilirken, obez hastalar ve gürültü etkisinin azaltılması gereken hastalar için yüksek tüp voltajları önerilmektedir [14].

Yapılan çalışmalarda, tüp akımı sabit tutulup, tüp voltajı değişimi yapıldığında dozun $\% 24,7$ ile \%45 arasında değiştiği bildirilmiştir [15-17]. Bizim çalışmamızda da kesit kalınlığı 0,625 mm iken $120 \mathrm{kVp}$ 'den $100 \mathrm{kVp}$ ye düşüldüğünde dozun 50,100, 200 ve 400 mAs akım değerleri için sırasıyla \%48,2, \%49,4, \%49,9 ve \%76,1 oranında düştüğü bulunmuştur.

Shah vd. [18] pediatrik hastalarda tüp akım değerinin azaltılmasının görüntü kalitesinde önemli bir etkiye sebep olmadığını bildirmişlerdir. Kranial CT çekimlerinde tüp akımlarının 90-130 mAs'den 76-90 mAs aralığına düşürülmesinin etkisin olmayacağını göstermişlerdir. Frush vd. [19] abdominal pediatrik CT çekimlerinde düşük tüp akımı değerinin kullanımının çözünürlük üzerine etkisi incelemiştir. $80 \mathrm{mAs}$ ve altındaki akım değerlerinde çözünürlüğün azalmasının belirgin olduğunu bulmuşlardır. Bununla beraber, Lucaya vd. [20], daha düşük tüp akımı değerlerinde (34-50 mA) kabul edilebilir bir görüntü kalitesine sahip olabileceğini öne sürmüşlerdir. Kamel vd. [21] ise 80-240 mAs arasında görüntü kalitesi veya tanı koyma aşamasında anlamlı bir fark olmadığını bulmuşlardır. Bizim çalışmamızda, artan akım değerinin tüm kesit kalınlığı ve voltaj değerleri için dozu arttırdığı gözlenmiştir. Örneğin, $400 \mathrm{mAs}$ akım değerinde doz artışı bütün kesit kalınlıklarında 100 mAs'a göre kafa fantomu için yaklaşık 7 kat artarken, vücut fantomu için, yaklaşık 3,7 kat artmaktadır. 
Saini vd. [22] kesit kalınlığının azaltılması durumunda, gürültü etkisini dengeleyebilmek için radyasyon dozunun arttırılması gerektiği bildirilmiştir. Konuyla ilgi çok fazla çalışma olmamakla beraber bizim çalışmamızda, $100 \mathrm{mAs}$ ve $80 \mathrm{kVp}$ için kesit kalınlığı artışı ile $(0,625 \mathrm{~mm}-10 \mathrm{~mm})$ kafa fantomu için $\% 15,8$ iken vücut fantomu için $\% 17,9$ oranında dozun azaldığ bulunmuştur.

Sonuç olarak, yukarıda bahsedilen çalışmalar sonucunda, iyi bir CT görüntüsünün yüksek dozlara gereksinim duymadığı açıktır. Tüp voltajının ve akımının ayarlanması ile görüntü kalitesinden ödün vermeden alınan doz azaltılabilir. Çalışma sonuçlarından da görüldüğü gibi, literatürle uyumlu olarak, doz artışı akım ve voltaj değerini arttırdıkça yükselmektedir. Özellikle kafa fantomu örneğindeki gibi küçük ve zayıf hastalarda doz artışı daha fazladır. Literatürde çok fazla çalışma bulunmamasına rağmen çalışmada kesit kalınlığı arttıkça doz düşmektedir. Fakat, özellikle kanser tanılı hastalarda, kesit kalınlığını küçük almak radyoterapi planlamasında, hedef hacmi tanımlamakta oldukça faydalı olabileceği düşünülmektedir.

\section{Teșekkür}

Bu çalışma, Buket Çeçen'in Yüksek lisans tez çalışması olup Akdeniz Üniversitesi BAPK FYL-2019-4804 nolu proje ile desteklenmiştir.

\section{Kaynakça}

[1] S. Reynolds, “The downside of Diagnostic Imaging," NCI Cancer Bulletin, 7(2), 8-9, 2010.

[2] UNSCEAR, "Sources, effects and risks of ionization radiation," UNSCEAR Report 2013 Vol. I, Report to General Assembly, New York, 2013, pp. 1-19.

[3] AAPM, "The measurement, reporting, and management of radiation dose in CT," AAPM Report No. 96, Report of AAPM Task Group 23 of the Diagnostic Imaging Council CT Committee, College Park, 2008, pp. 27-35.

[4] AAPM, "Site specific dose estimates (SSDE) in pediatric and adult body CT examinations," AAPM Report No. 204, Report of AAPM Task Group 204 of AAPM, College Park, 2011, pp. 41-56.

[5] AAPM, "Comprehensive methodology for the evaluation of radiation dose in X-ray computed tomography," AAPM Report No. 111, Report of AAPM Task Group 111: The future of CT dosimetry, College Park, 2010, pp. 21-23.

[6] J. A. Bauhs, T. J. Vrieze, A. N. Primak, M. R. Bruesewitz, and C. H. McCollough, "CT dosimetry: comparison of measurement techniques and devices," Radiographics, 28 (1), 245-253, 2008.

[7] M. J. Siegel, B. Schmidt, D. Bradley, C. Suess, and C. Hildebolt, "Radiation dose and image quality in pediatric CT: effect of technical factors and phantom size and shape," Radiology, 233 (2), 515$522,2004$.

[8] J. J. DeMarco, C. H. Cagnon, D. D. Cody, D. M. Stevens, C. H. McCollough, M. Zankl, E. Angel, and M. F. McNitt-Gray, "Estimating radiation doses from multidetector CT using Monte Carlo simulations: effects of different size voxelized patient models on magnitudes of organ and effective dose," Phys. Med. Biol., 52 (9), 2583-2597, 2007.

[9] A. C. Turner, M. Zankl, J. J. DeMarco, C. H. Cagnon, D. Zhang, E. Angel, D. D. Cody, D. M. Stevens, C. H. McCollough, and M. F. McNitt-Gray, "The feasibility of a scanner-independent technique to estimate organ dose from MDCT scans: using CTDIvol to account for differences between scanners," Med. Phys., 37 (4), 1816-1825, 2010.

[10] S. M. Lee, W. Lee, J. W. Chung, E.-A. Park, and J. H. Park, "Effect of kVp on image quality and accuracy in coronary CT angiography according to patient body size: a phantom study," Int. J. of Cardiovascular Imaging, 29, 83-91, 2013.

[11] Q. Li, H. Yua, L. Zhang, L. Fana, and S.-Y. Liu, "Combining low tube voltage and iterative reconstruction for contrast-enhanced CT imaging of the chest - Initial clinical experience," Clinical Radiology, 68 (5), 249-253, 2013.

[12] Z. Szucs-Farkas, C. Schaller, S. Bensler, M. A. Patak, P. Vock, and S. T. Schindera, "Detection of pulmonary emboli with $\mathrm{CT}$ angiography at reduced radiation exposure and contrast material volume: comparison of $80 \mathrm{kVp}$ and $120 \mathrm{kVp}$ protocols in a matched cohort," Investigative Radiology, 44 (12), 793-799, 2009.

[13] L. Schimmoller, R. S. Lanzman, S. Dietrich, J. Boos, P. Heusch, F. Miese, G. Antoch, and P. Kropil, "Evaluation of automated attenuation-based tube potential selection in combination with 
organ-specific dose reduction for contrast-enhanced chest CT examinations," Clinical Radiology, 69 (7), 721-726, 2014.

[14] H. W. Goo, "CT radiation dose optimization and estimation: an update for radiologists," Korean Journal of Radiology, 13 (1), 1-11, 2012.

[15] C. M. Heyer, P. S. Mohr, S. P. Lemburg, S. A. Peters, and V. Nicolas, "Image quality and radiation exposure at pulmonary CT angiography with $100-$ or $120-\mathrm{kVp}$ protocol: prospective randomized study," Radiology, 245 (2), 577-583, 2007.

[16] A. Sodickson, and M. Weiss, "Effects of patient size on radiation dose reduction and image quality in low-kVp CT pulmonary angiography performed with reduced IV contrast dose", Emerg. Radiol., 19 (5), 437-445, 2012.

[17] P. Bjorkdahl, and U. Nyman, "Using 100- instead of 120-kVp computed tomography to diagnose pulmonary embolism almost halves the radiation dose with preserved diagnostic quality," Acta Radiologica, 51 (3), 260-270, 2010.

[18] R. Shah, A. K. Gupta, M. M. Rehani, A. K. Pande, and S. Mukhopadhyay, "Effect of reduction in tube current on reader confidence in pediatric computed tomography," Clinical Radiology, 60, 2242312005.

[19] D. P. Frush, C. C. Slack, C. L. Hollingsworth, G. S. Bisset, L. F. Donnelly, J. Hsieh, T. LavinWensell, and J. R. Mayo, "Computer simulated radiation dose reduction for abdominal multidetector CT of pediatric patients," AJR Am. J. Roentgenol. 2002;179: 1107-13.

[20] J. Lucaya, J. Piqueras, P. Garcia-Pena, G. Enriquez, M. Garcia-Macias, and J. Sotil, "Low-dose high resolution CT of the chest in children and young adults: dose, cooperation, artifact incidence, and image quality," AJR Am. J. Roengenol., 175 (4), 985-992, 2000.

[21] I. R. Kamel, R. J. Hernandez, J. E. Martin, A. E. Schlesinger, L. T. Niklason, and K. E. Guire, "Radiation dose reduction of the pediatric pelvis," Radiology, 190 (3), 683-687, 1994.

[22] S. Saini, "Multi-detector row CT: principles and practice for abdominal applications," Radiology, 233, 323-327, 2004. 